Published in final edited form as:

J Comp Eff Res. 2015 January ; 4(1): 27-35. doi:10.2217/cer.14.53.

\title{
Revisiting the washout period in the incident user study design: why 6-12 months may not be sufficient
}

\author{
Andrew W Roberts ${ }^{\star}, 1,2$, Stacie B Dusetzina ${ }^{1,2,3,4}$, and Joel F Farley ${ }^{1}$ \\ 1Division of Pharmaceutical Outcomes \& Policy, UNC Eshelman School of Pharmacy, NC, USA \\ ${ }^{2}$ Cecil G Sheps Center for Health Services Research, University of North Carolina, Chapel Hill, \\ NC, USA \\ ${ }^{3}$ Department of Health Policy \& Management, UNC Gillings School of Global Public Health, NC, \\ USA \\ ${ }^{4}$ Lineberger Comprehensive Cancer Center, University of North Carolina, Chapel Hill, NC, USA
}

Abstract

Aims-The purpose of this study was to describe how washout period duration affects the size and accuracy of retrospective incident user cohorts.

Materials \& methods-MarketScan commercial claims data from 2007 to 2010 were used and included adults with an antihyperlipidemic, antidiabetic or antidepressant claim in 2010. Incident user cohorts using 3-, 6-, 12-, 24- and 36-month washouts were created and changes in sample size and incident user misclassification were described.

Results \& conclusion-The 6- and 12-month washouts excluded 75 and $85 \%$ of the samples, respectively. Half of subjects in the 6-month washout cohorts were actually prevalent users, and the 12-month washout period resulted in 30\% misclassified. Using common washout periods of $6-$ 12 months may insufficiently address prevalent user bias in large commercial claims databases.

\section{Keywords}

comparative effectiveness research; incident user design; methods; pharmacoepidemiology; research design; secondary databases; selection bias

\footnotetext{
() 2015 Future Medicine Ltd

*Author for correspondence: Tel.: +1 919966 7557, Fax: +1 919966 8486, awroberts@unc.edu.

For reprint orders, please contact: reprints@ futuremedicine.com
}

Findings from this study were presented at the 2014 American Pharmacists Association Annual Meeting, where it won the APhAAPRS ESAS Best Paper Podium Award. This study was also presented orally at the 2014 NRSA Trainees' Conference in June 2014 in San Diego, CA.

Financial \& competing interests disclosure: The authors have no other relevant affiliations or financial involvement with any organization or entity with a financial interest in or financial conflict with the subject matter or materials discussed in the manuscript apart from those disclosed.

No writing assistance was utilized in the production of this manuscript.

Ethical conduct of research: The authors state that they have obtained appropriate institutional review board approval or have followed the principles outlined in the Declaration of Helsinki for all human or animal experimental investigations. In addition, for investigations involving human subjects, informed consent has been obtained from the participants involved. 


\section{Background}

In recent decades, the fields of pharmaceutical outcomes, pharmacoepidemiology, pharmacovigilance and comparative effectiveness research have increasingly relied on observational cohort study methodologies employed in large administrative claims data sets. $[1,2]$ The primary challenge of these studies lies in addressing selection bias stemming from the lack of randomization of study subjects to treatment [3]. Prevalent user bias is one common form of selection bias and confounding that occurs when observational cohort studies include subjects with prior exposure to the medication therapy being investigated [4,5]. Prevalent user bias typically manifests through inflated estimates of medication benefits and safety because studies including prevalent users in their analyses have disproportionately selected for patients with demonstrated success on therapy [6-8]. For example, a recent meta-analysis of observational cohort studies investigating the association of statin use and mortality found that the studies designed to mitigate prevalent user bias estimated a $23 \%$ mortality reduction, whereas the studies that failed to address prevalent user bias reported a $46 \%$ mortality reduction attributable to statin use, inappropriately doubling the true estimates of benefit [6].

Researchers reduce prevalent user bias by restricting samples to new, or incident, users of study medications [9]. This is achieved through identifying and excluding patients that exhibit previous use of the study medication in claims data during a pre-index date "washout period' $[4,5]$. Selection of the washout period duration in an incident user design presents clear trade-offs for observational researchers. A longer washout period assures greater internal validity due to more thorough assessment of prevalent use [10], but these longer washout periods reduce generalizability and estimate precision, through the loss of sample size with increasing restrictions [11]. Although certain factors, such as the length of time a drug has been on the market and data availability, can help inform the washout duration decision, a washout period legacy standard of 6-12 months has developed among observational researchers $[11,12]$. However, little evidence exists to explicitly substantiate the use of 6-12-month washout periods in claims-based incident user studies or to inform appropriate washout period selection based for a specific research question [13,14].

The purpose of this study was to describe the effect of implementing washout period criteria of increasing duration on sample size and accuracy of retrospective, incident user cohorts across three condition-centric groups: antihyperlipidemic, antidiabetic and antidepressant users. The implications of washout period duration are evaluated in these three clinical areas, as they are highly prevalent medically-managed conditions with varying clinical courses and presentations [15-19].

\section{Materials \& methods}

\section{Data source}

Truven Health Analytics MarketScan Commercial Claims and Encounters data from 2007 to 2010 were used. MarketScan captures health insurance enrollment information, as well as inpatient, outpatient and pharmacy utilization data for roughly 20 million private health insurance beneficiaries annually from approximately 100 commercial payers [20]. 


\section{Study subject selection}

Adults aged 18-64 years with at least one outpatient prescription claim in 2010 for drugs approved in the USA to treat hyperlipidemia, diabetes or depression were included. Antihyperlipidemic medications included HMG-CoA reductase inhibitors (statins), ezetimibe, bile acid sequestrants, fibric acid derivatives, prescription-only niacin preparations and prescription-only omega-3-acid ethyl esters [17,21]. Antidiabetic medications included metformin, sulfonylureas, thiazolidinediones, dipeptidyl peptidase- 4 inhibitors, meglitinides, alpha-glucosidase inhibitors, and injectable insulin and incretin mimetic formulations [16,22]. Antidepressant medications included selective serotonin reuptake inhibitors, serotonin-norepinephrine reuptake inhibitors, tricyclics, bupropion, maprotiline, monoamine oxidase inhibitors and serotonin antagonist reuptake inhibitors [23]. Combination products with at least one of the listed study medications were also included.

\section{Study cohort definition}

The index fill was the first fill date for a qualifying medication in one of the three study cohorts in calendar year 2010. Individuals could be included in multiple disease-state-centric cohorts and the index date was allowed to vary between cohorts for such individuals. For each of the three cohorts, a series of five washout periods of increasing length $-3,6,12,24$ and 36 months - was used to assess prevalent use and create increasingly restrictive incident user cohorts (Figure 1). A treatment-naive definition of incident use was used, in which having at least one prescription claim for any cohort-specific study medication in the washout period, regardless of whether or not it matched the index drug or index drug class, constituted prevalent use. For example, an antihyperlipidemic user whose index medication was simvastatin would be considered a prevalent user if, in the given washout period, they had a claim for simvastatin, another statin or any other antihyperlipidemic agent. This is the strictest definition of prevalent use and is commonly employed in comparative effectiveness studies of pharmacotherapies, in which subjects are required to have never been treated for the condition under study to ensure similar baseline risks of the study outcome across groups. Inclusion in each of the incident user cohorts also required continuous insurance enrollment for the duration of the given washout period, defined by having a gap in coverage no greater than 7 days. This is necessary in incident user designs to ensure complete assessment of a subject's medication use for the entire washout period. A sensitivity analysis allowing a 30-day gap in insurance coverage was conducted; the results were unchanged, so the findings are presented using a 7-day coverage gap.

\section{Analysis}

Changes in sample size were described for each condition-centric cohort as the increasingly restrictive washout criteria were applied. The singular effect of the continuous prior enrollment requirement on sample size was also examined to understand its impact separately from incident use criteria. Last, for each of the hyperlipidemia, diabetes and depression cohorts the rate of incident user misclassification across washout durations was described. To assess this, we restricted our analysis to subjects with a full 36 months of continuous insurance enrollment prior to their index fill date. Among these individuals, subjects meeting the 36-month washout criteria were considered true incident users.

J Comp Eff Res. Author manuscript; available in PMC 2015 November 01. 
Washout periods of 3, 6, 12 and 24 months were applied to those with 36 months of continuous enrollment. Additional subjects included in incident user cohorts created from shorter washout periods beyond those included in the true incident user cohort were considered misclassified due to the washout period's inability to identify known prevalent use between 36 months pre-index date and the start of the washout period. SAS 9.3 (Cary, NC) was used for all analyses. Approval for this study was obtained from the University of North Carolina at Chapel Hill Institutional Review Board.

\section{Results}

We identified 3,477,743 antihyperlipidemic, 1,455,651 antidiabetic, and 3,468,137 antidepressant users with an index fill in calendar year 2010. Sample size loss in each disease-state cohort due to continuous enrollment and the washout period criteria, is reported in Table 1.

Requiring 3 months of continuous insurance enrollment prior to the index fill date excluded roughly $25 \%$ of subjects with an index medication fill across the three disease states, with 6 and 12 months of continuous enrollment excluding around 30 and $35 \%$ of subjects, respectively (Table 1). The antidepressant user cohort was slightly more susceptible to sample loss due to continuous enrollment requirements; $71.2 \%$ of the original antidepressant user cohort was excluded with a 36-month continuous enrollment requirement compared with 65.6 and $65.7 \%$ for antihyperlipidemic and antidiabetic users, respectively.

Applying a 3-month washout period, in addition to the continuous enrollment requirement, resulted in loss of 54.1, 60.5 and $56.1 \%$ of subjects with any antihyperlipidemic, antidiabetic and antidepressant use in 2010, respectively. The legacy standard washout periods of 6 and 12 months created incident user cohorts comprised only $22-27 \%$ and $13-17 \%$ of original subjects with an index antihyperlipidemic, antidiabetic and antidepressant prescription fill, respectively. Only $5 \%$ of subjects in each original cohort met the incident user criteria based on the most restrictive washout period of 36 months.

The observed changes in sample size across the 3, 6, 12, 24 and 36-month look-back periods are visualized in Figure 2A-C, which depict the relative impact of the continuous insurance enrollment requirement on sample size loss for each drug class of interest. A lack of continuous insurance enrollment was the cause of exclusion for roughly $40 \%$ of those excluded from incident user cohorts based on 3, 6 and 12-month washout periods. At longer washout periods of 24 and 36 months, 53 and $69 \%$ of the observed sample loss was due to the continuous enrollment requirement, respectively. The effect of continuous enrollment requirement was slightly greater in the antidepressant cohort. Over $45 \%$ of those excluded based on the 3- to 12-month washout periods were excluded due to insurance coverage gaps, as opposed to the incident use criteria. Of all subjects excluded by the 24 and 36-month washout periods, nearly two-thirds and three-quarters, respectively, were attributed to failing to meet the continuous enrollment requirement.

Figure 3 depicts the extent of incident user misclassification across washout period durations among the 1,195,528 antihyperlipidemic, 474,092 antidiabetic and 997,962 antidepressant 
users with 36 months of continuous enrollment prior to their index fill. In each disease state, misclassification of incident users increased steadily as washout periods decreased. When using a 12-month washout period, $25.5 \%$ of the antidiabetic and nearly one-third of the antihyperlipidemic and antidepressant users identified as incident users actually had prevalent use at some point during the entire 36-month pre-index period. Decreasing the washout period to 6 months resulted in an incident user cohort in which half of the subjects had prevalent use while $67-77 \%$ of those identified as incident users using a 3-month washout period were misclassified.

Out of the three disease states, antihyperlipidemic users were most likely to be misclassified in the 3-, 6- and 12-month washouts, and antidepressant incident users experienced the greatest misclassification at the 24-month washout period. Antidiabetic users were generally least likely to be misclassified as incident users across washout periods. However, misclassification trends were comparable across disease states.

\section{Discussion}

The effect of using washout periods of increasing duration on the size and accuracy of antihyperlipidemic, antidiabetic and antidepressant incident user cohorts in a large administrative claims database was described. In general, the observed trends in sample size loss and incident user misclassification across washout periods were comparable across disease states with varying clinical courses and presentations.

Substantial and rapid loss of sample size was found with commonly used continuous enrollment and washout period criteria of 6 and 12 months, which resulted in exclusion of about 75 and $85 \%$ of initially qualifying drug users, respectively. Continuous enrollment requirements were responsible for about $40-45 \%$ of observed sample size loss in the 3 - to 12-month washout cohorts and over half to two-thirds of the sample size loss in the most restrictive washout cohorts.

Study findings related to incident user misclassification highlight serious concerns over the ability of 6- and 12-month washout periods to accurately identify incident users. Not only did the 6-month continuous enrollment and washout period result in a loss of three-quarters of the original sample across the disease states, but one out of every two subjects included in the incident user cohorts actually had prior use at some point between 6 and 36 months preceding the index fill. Extending the washout period to 12 months still resulted in the creation of incident user cohorts in which one-third of included subjects were incorrectly classified as incident users, while losing an additional $10 \%$ of the original sample. This suggests that observational incident user studies employing commonly used washout periods of 6-12 months likely suffer from notable residual prevalent user bias.

Incident user study designs require careful consideration of the consequences of the washout period duration selection. Shorter washout periods of 3-6 months may be necessary in the context of a priori concerns about sample size, limited data availability or when investigating newly-approved therapies. However, in light of these results showing that washout periods of 12 months or less may be insufficient for accurately identifying 
prevalent users, researchers implementing incident user designs should consider employing washout periods greater than 12 months to better ensure their cohorts contain treatmentnaive patients. Doing so could drastically reduce the threat of prevalent user bias while still allowing for adequately powered analyses, especially in studies involving very large data sets and/or highly prevalent pharmacotherapies. For example, extending the washout period in our antihyperlipidemic cohort from 12 to 24 months resulted in excluding only an additional $5 \%$ of the original sample of 3.5 million users, while reducing incident user misclassification from $33 \%$ to a little over $10 \%$. At a minimum, researchers should conduct sensitivity analyses using varied washout period durations to assess the robustness of their study estimates in the face of potential residual prevalent user bias. While the goals of the study will dictate the relative importance of using longer washout periods, studies that require treatment naive patients may only be internally valid if restricted to the small sample with long washout periods [24,25]. Given the relative importance of internal validity over generalizability, the loss in sample is a reasonable trade-off.

Findings from our study should be interpreted in light of key limitations. First, a treatmentnaive definition of incident use was employed, in which a subject was deemed a prevalent user if they had filled a prescription for any medication indicated for the specific disease state in question. This approach may not be appropriate for all research questions; for example, in an observational cohort study investigating a drug-classspecific adverse effect, it would likely be more appropriate to define prevalent use as any prior exposure solely to medications in that specific drug class. Similarly, it may be appropriate to include individuals who had distant prior, short-term use of a drug depending on the goals of the study. Second, selection of an exposed cohort was the focus of this study, rather than selection of controls. It is possible that there may be differential detection of prevalent use among selected treatment and control groups for comparative effectiveness studies, which may introduce bias. Last, this study was conducted among privately insured adults. The findings may not generalize to observational incident user studies conducted in other patient populations, such as Medicaid patients, who are known to experience more frequent gaps in insurance enrollment [26].

\section{Conclusion}

The effect of washout period duration on the size and accuracy of incident user cohorts in a large commercial insurance claims data set was examined. The findings emphasize the need for observational researchers to recognize that commonly used 6- to 12-month washout periods may insufficiently control for prevalent user bias. Researchers employing incident user study designs should carefully consider the duration of their washout period, utilize a washout period greater than 12 months when possible, and at the very least, conduct sensitivity analyses around their selected washout period to examine its influence on the specific population and outcomes being studied. There is a clear need to better understand the effects of incident use criteria on the results and generalizability of observational incident user studies in large claims data sets, especially considering the increasing reliance on these study methodologies to contribute to evidence-based clinical and regulatory decision-making.

J Comp Eff Res. Author manuscript; available in PMC 2015 November 01. 


\section{Future perspective}

The role of observational research methods in large data sets, including incident user study designs, will continue to expand in the field of comparative effectiveness research of pharmacotherapies. During this time, researchers will continue to explore and employ rigorous research design and analytic strategies that improve the control of selection bias. This will be necessary not only to ensure that estimates of association between medications and outcomes reflect the truth to the greatest extent possible, but also to foster a strong sense of legitimacy in the contributions of observational comparative effectiveness research to the clinical evidence-base among key policy, provider, payer and patient stakeholders.

\section{Acknowledgments}

The authors would like to thank MA Brookhart, associate professor of epidemiology in the UNC Gillings School of Global Public Health, for his guidance related to this investigation.

The authors would like to acknowledge the following funding sources that provided salary support for the investigators: NRSA T32 Postdoctoral Fellowship from AHRQ sponsored by the Cecil G Sheps Center for Health Services Research, UNC Chapel Hill, Grant No. 5T32 HS000032 (Roberts); NIH Building Interdisciplinary Research Careers in Women's Health (BIRCWH) K12 Program and the North Carolina Translational and Clinical Sciences Institute (UL1TR001111) (Dusetzina). J Farley has received prior consulting support from Daiichi-Sankyo and Novartis for entirely unrelated research.

\section{References}

Papers of special note have been highlighted as:

• of interest; $\bullet$ of considerable interest

1. Schneeweiss S, Avorn J. A review of uses of health care utilization databases for epidemiologic research on therapeutics. J Clin Epidemiol. 2005; 58(4):323-337. [PubMed: 15862718]

2. Patient-Centered Outcomes Research Institute. National Priorities for Research and Research Agenda. 2012. www.pcori.org

3. Cox E, Martin BC, Van Staa T, Garbe E, Siebert U, Johnson ML. Good research practices for comparative effectiveness research: approaches to mitigate bias and confounding in the design of nonrandomized studies of treatment effects using secondary data sources: the International Society for Pharmacoeconomics and Outcomes Research Good Research Practices for Retrospective Database Analysis Task Force Report--Part II. Value Health. 2009; 12(8):1053-1061. [PubMed: 19744292]

4. Brookhart MA, Sturmer T, Glynn RJ, Rassen J, Schneeweiss S. Confounding control in healthcare database research: challenges and potential approaches. Med Care. 2010; 48(6 (Suppl.)):S114 S120. [PubMed: 20473199]

5••. Ray WA. Evaluating medication effects outside of clinical trials: new-user designs. Am J Epidemiol. 2003; 158(9):915-920. Key paper describing, in detail, the impact of prevalent user bias in observational studies and the role of the incident user design in mitigating this bias. [PubMed: 14585769]

6•. Danaei G, Tavakkoli M, Hernan MA. Bias in observational studies of prevalent users: lessons for comparative effectiveness research from a meta-analysis of statins. Am J Epidemiol. 2012; 175(4):250-262. Excellent meta-analysis quantifying the impact of prevalent user bias in observational studies of the association between statins and mortality. [PubMed: 22223710]

7•. Maciejewski ML, Bryson CL, Wang V, Perkins M, Liu CF. Potential bias in medication adherence studies of prevalent users. Health Serv Res. 2013; 48(4):1468-1486. Study examining the effect of varied definitions of incident use on estimates of medication adherence. [PubMed: 23402554]

J Comp Eff Res. Author manuscript; available in PMC 2015 November 01. 
8. Schneeweiss S, Patrick AR, Sturmer T, et al. Increasing levels of restriction in pharmacoepidemiologic database studies of elderly and comparison with randomized trial results. Med Care. 2007; 45(10 (Suppl. 2)):S131-S142. [PubMed: 17909372]

9. Perrio M, Waller PC, Shakir SA. An analysis of the exclusion criteria used in observational pharmacoepidemiological studies. Pharmacoepidemiol Drug Saf. 2007; 16(3):329-336. [PubMed: 16741894]

10•. Brunelli SM, Gagne JJ, Huybrechts KF, et al. Estimation using all available covariate information versus a fixed look-back window for dichotomous covariates. Pharmacoepidemiol Drug Saf. 2013; 22(5):542-550. Simulation study demonstrating the eduction in bias that occurs as researchers utilize more pre-index patient data. [PubMed: 23526818]

11••. Johnson ES, Bartman BA, Briesacher BA, et al. The incident user design in comparative effectiveness research. Pharmacoepidemiol Drug Saf. 2013; 22(1):1-6. Comprehensive white paper on current best practices for use of the incident user study design. [PubMed: 23023988]

12. Ray WA, Stein CM, Daugherty JR, Hall K, Arbogast PG, Griffin MR. COX-2 selective nonsteroidal antiinflammatory drugs and risk of serious coronary heart disease. Lancet. 2002; 360(9339):1071-1073. [PubMed: 12383990]

13. Abbas S, Ihle P, Koster I, Schubert I. Estimation of disease incidence in claims data dependent on the length of follow-up: a methodological approach. Health Serv Res. 2012; 47(2):746-755. [PubMed: 21958217]

14- Gardarsdottir H, Heerdink ER, Egberts AC. Potential bias in pharmacoepidemiological studies due to the length of the drug free period: a study on antidepressant drug use in adults in the Netherlands. Pharmacoepidemiol Drug Saf. 2006; 15(5):338-343. Investigation of washout period implications in a large Dutch national healthcare system database. [PubMed: 16496430]

15. Kessler RC, Berglund P, Demler O, et al. The epidemiology of major depressive disorder results from the National Comorbidity Survey Replication (NCS-R). JAMA. 2003; 289(23):3095-3105. [PubMed: 12813115]

16. Nathan DM, Buse JB, Davidson MB, et al. Medical management of hyperglycemia in type 2 diabetes a consensus algorithm for the initiation and adjustment of therapy: a consensus statement of the American Diabetes Association and the European Association for the Study of Diabetes. Diabetes Care. 2009; 32(1):193-203. [PubMed: 18945920]

17. National Cholesterol Education Program Expert Panel on Detection E, Treatment of High Blood Cholesterol In Adults. Third Report of the National Cholesterol Education Program (NCEP) Expert Panel on Detection, Evaluation, and Treatment of High Blood Cholesterol in Adults (Adult Treatment Panel III) final report. Circulation. 2002; 106(25):3143-3421. [PubMed: 12485966]

18. Satyanarayana S, Enns MW, Cox BJ, Sareen J. Prevalence and correlates of chronic depression in the Canadian community health survey: mental health and well-being. Can J Psychiatry. 2009; 54(6):389-398. [PubMed: 19527559]

19. Schneeweiss S, Patrick AR, Solomon DH, et al. Variation in the risk of suicide attempts and completed suicides by antidepressant agent in adults: a propensity score-adjusted analysis of 9 years' data. Arch Gen Psychiatry. 2010; 67(5):497-506. [PubMed: 20439831]

20. Truven Health Analytics. Better Understand Health Economics and Treatment Outcomes: Data Databases and Online Tools. 2013. http://truvenhealth.com

21. Last AR, Ference JD, Falleroni J. Pharmacologic treatment of hyperlipidemia. Am Fam Physician. 2011; 84(5):551-558. [PubMed: 21888306]

22. Garber AJ, Abrahamson MJ, Barzilay JI, et al. AACE comprehensive diabetes management algorithm 2013. Endocr Pract. 2013; 19(2):327-336. [PubMed: 23598536]

23. American Psychiatric Association. Practice Guidelines for the Treatment of Patients With Major Depressive Disorder. 3rd2010. http://psychiatryonline.org

24. Berger VW. Pros and cons of permutation tests in clinical trials. Stat Med. 2000; 19(10):13191328. [PubMed: 10814980]

25. Grimes DA, Schulz KF. Bias and causal associations in observational research. Lancet. 2002; 359(9302):248-252. [PubMed: 11812579]

26. Sommers BD. Loss of health insurance among non-elderly adults in Medicaid. J Gen Intern Med. 2009; 24(1):1-7. [PubMed: 18810555]

J Comp Eff Res. Author manuscript; available in PMC 2015 November 01. 


\section{Executive summary}

\section{Background \& purpose}

- Observational comparative effectiveness research of pharmacotherapies often requires the incident user design, which reduces bias through the use of washout periods that identify and exclude subjects with prior use of the study medication.

- Researchers typically choose a 6- or 12-month washout period, despite limited evidence available to inform appropriate washout selection.

- The purpose of this study was to describe the effect of washout periods of increasing duration on sample size and accurate identification of incident users in a large commercial claims database.

\section{Materials \& methods}

- MarketScan commercial claims data for years 2007-10 were used.

- Adults with a prescription claim for an antihyperlipidemic, antidiabetic, and/or antidepressant in 2010 were included, and a series of treatment-naive incident user cohorts within each disease state was created using washout periods of 3,6 , 12, 24 and 36 months.

- Sample size loss across washout periods was described, as well as the singular effect of requiring subjects to have continuous insurance enrollment during the look-back period.

- The prevalence of misclassification in incident user cohorts across washout period durations was also described, by reporting the percentage of subjects that had prior exposure to a cohort medication preceding a given washout period.

\section{Results}

- Sample size dropped rapidly as washout periods increased, with roughly $85 \%$ of subjects excluded with a 12-month washout period.

- Lapses in continuous insurance enrollment in the data set were responsible for $40 \%$ of the observed sample loss in washout periods of a year or less.

- Half of subjects identified as incident users with a 6-month washout period actually had a prior claim for a drug indicated for that condition, and $30 \%$ of subjects included in the 12-month washout incident user cohort were misclassified.

\section{Discussion \& conclusion}

- Commonly used washout periods of 6-12 months may insufficiently control for prevalent user bias.

- Observational researchers employing an incident user design should consider using washout periods longer than 12 months when appropriate and possible. 
- At minimum, sensitivity analyses around washout period duration should be conducted in incident user designs to assess the robustness of study estimates in the face of possible residual prevalent user bias. 

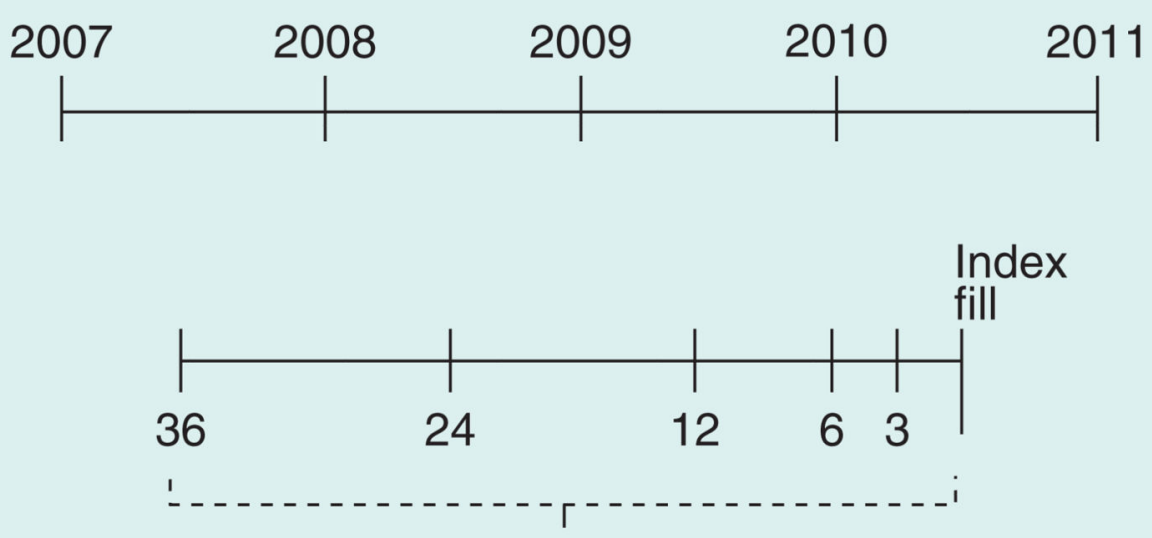

Washout period (months) PLUS continuous enrollment

Figure 1. Diagram depicting the creation of increasingly restrictive incident user cohorts using varied washout period durations in a single diseasestate-centric cohort

Figure 1 visualizes how the incident user cohorts were created with five washout periods of increasing duration anchored by the index prescription fill in 2010. 

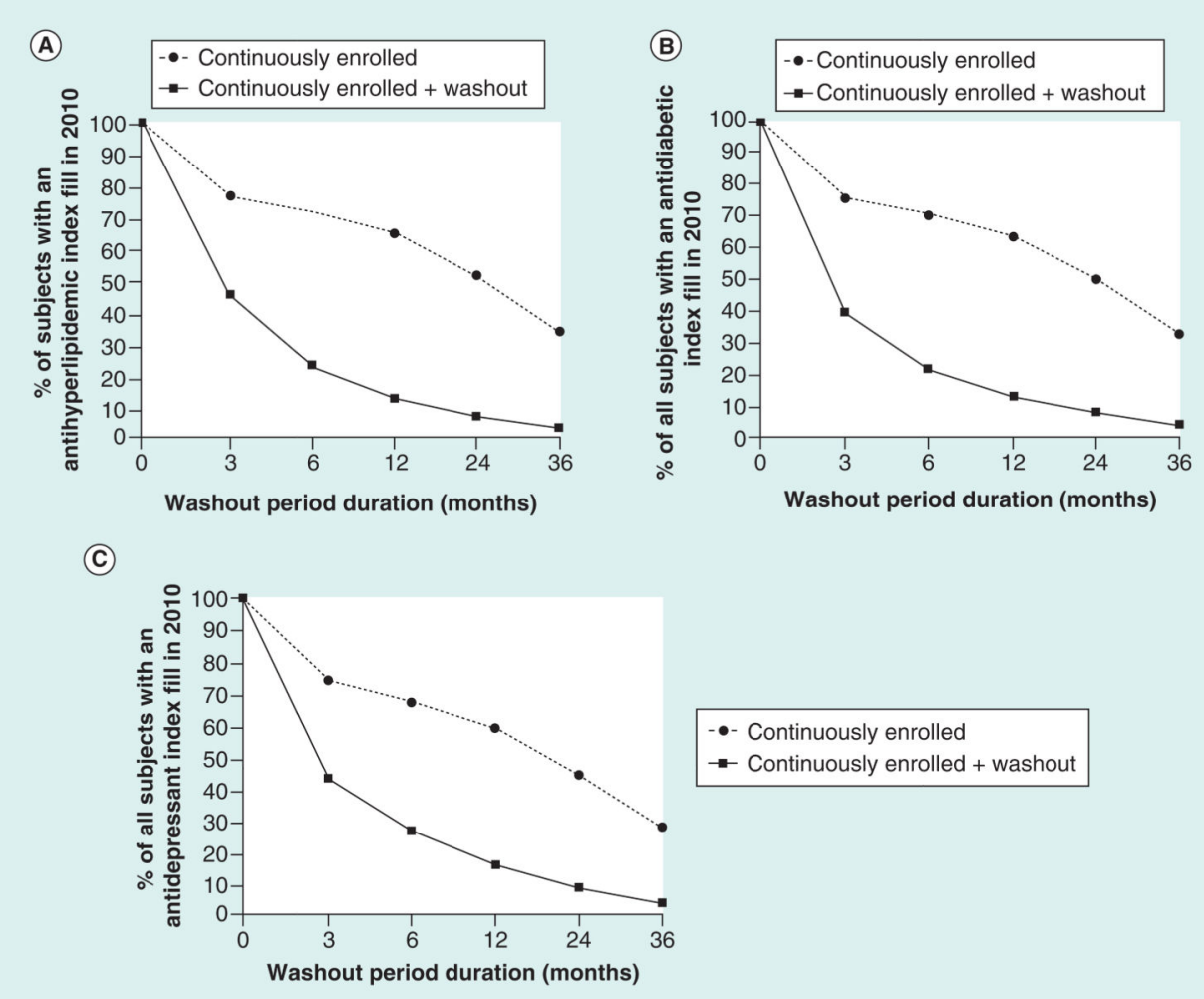

Figure 2. Incident user cohort sample size loss across washout periods, by disease state This figure depicts the proportion of patients with an index fill in 2010 meeting the continuous enrollment and continuous enrollment plus washout criteria across look-back periods of increasing duration. Panels (A), (B), and (C) represent incident user cohort size changes across look-back periods for antihyperlipidemic, antidiabetic, and antidepressant user cohorts, respectively. Note: There were a total of 3,477,753 antihyperlipidemic, 1,445,651 antidiabetic and 3,468,137 antidepressant users aged 18-64 years in calendar year 2010. 


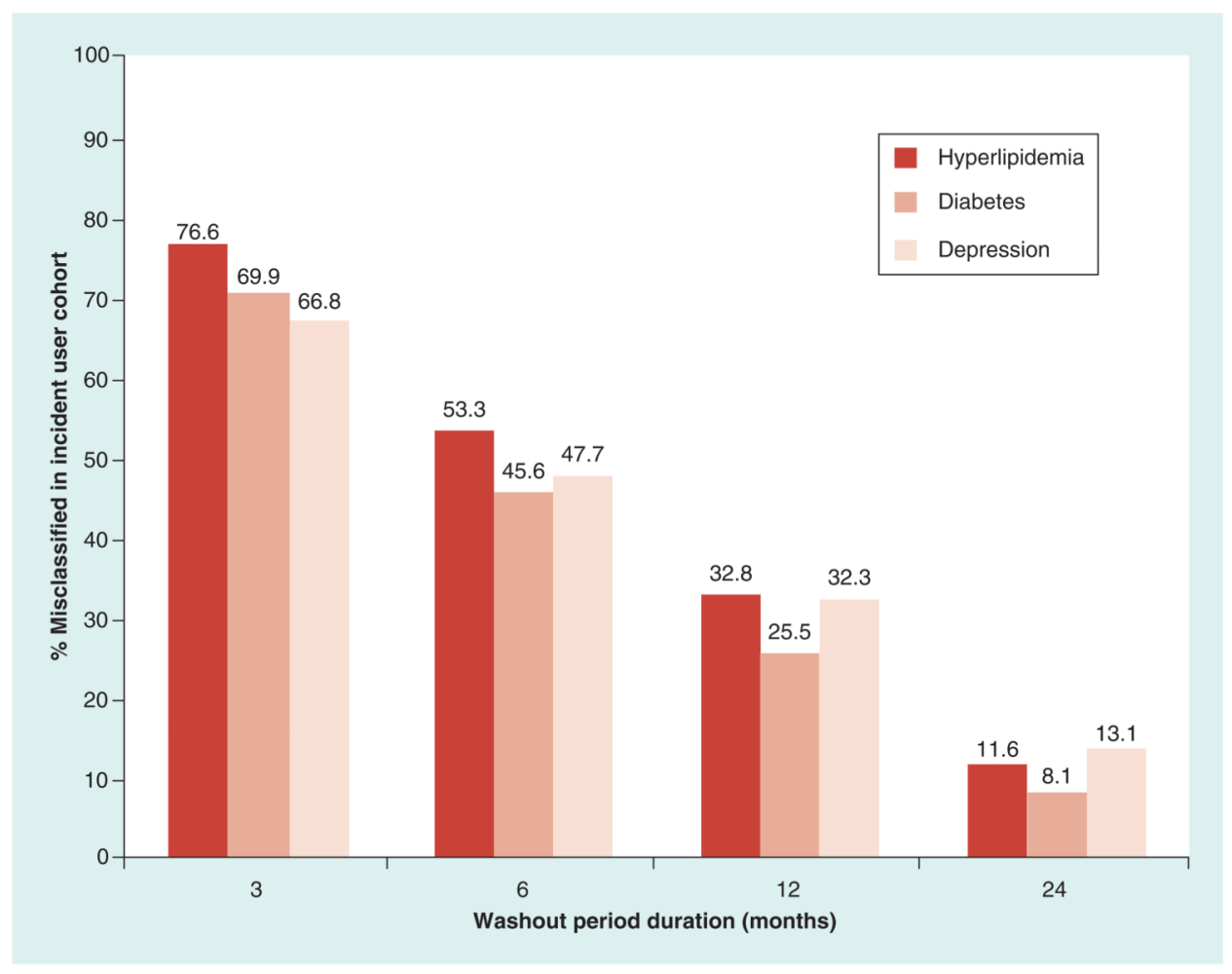

Figure 3. Misclassification of subjects as new users with increasingly restrictive washout periods among subjects with 36 months of continuous enrollment

The figure depicts that as the pre-index washout period duration decreases, the proportion of the incident user cohort comprised of subjects with prevalent use who were misclassified as incident users, increases steadily. Note: A total of 1,195,528, 474,092 and 997,962 study subjects had 36 months of continuous enrollment prior to the index fill for the hyperlipidemia, diabetes and depression cohorts, respectively. Study subjects meeting the 36-month washout period criterion were considered true incident users. Reported numeric values represent percentage of those included in the incident user cohort that were actually prevalent users. 


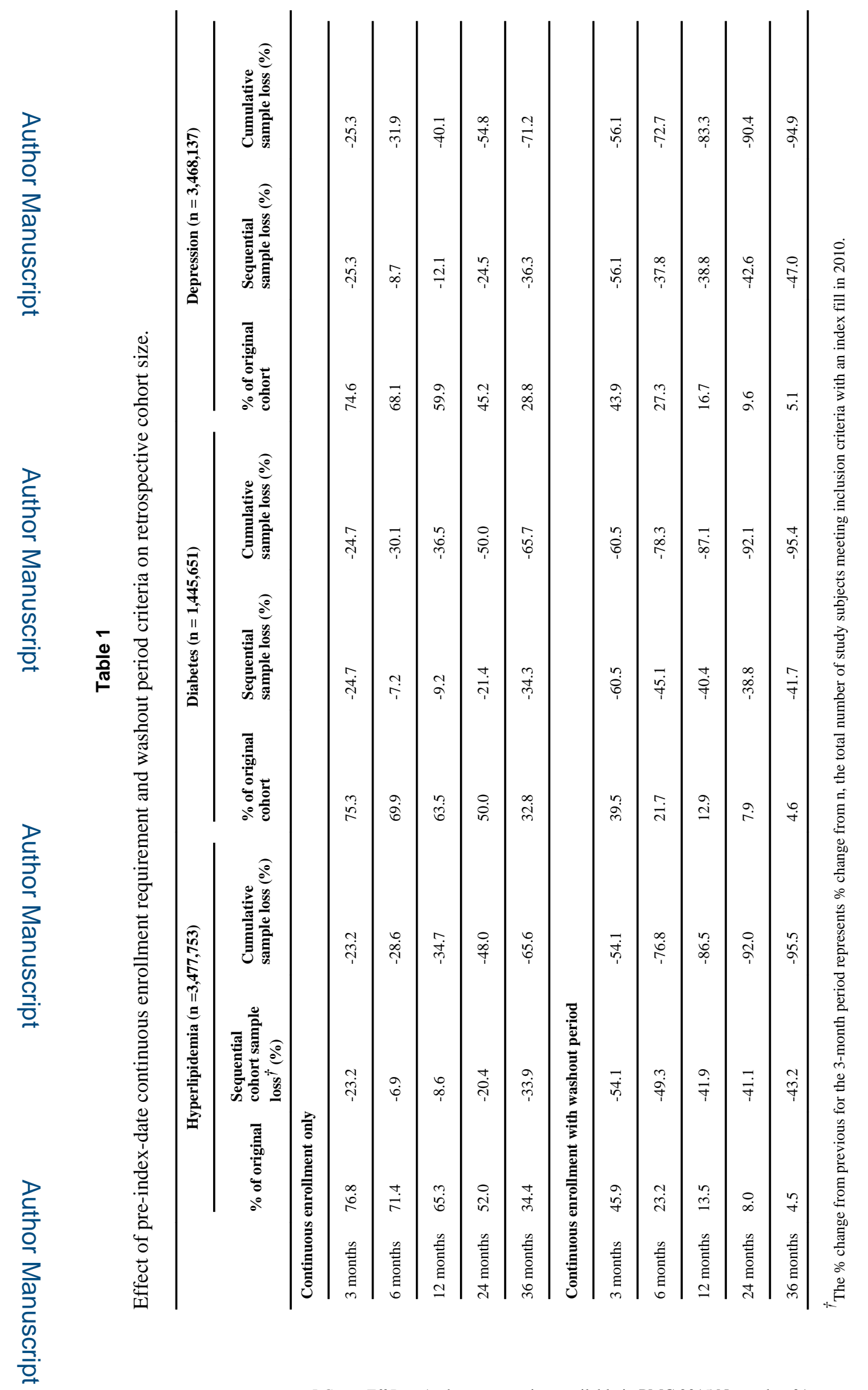

J Comp Eff Res. Author manuscript; available in PMC 2015 November 01. 